\title{
Possible errors in the article "Potential benefits of salvage intratympanic dexamethasone injection in profound idiopathic sudden sensorineural hearing loss"
}

\author{
Xizheng Shan ${ }^{1} \cdot$ Entong Wang ${ }^{1}$ (i)
}

Received: 2 August 2020 / Accepted: 17 August 2020 / Published online: 25 August 2020

(c) Springer-Verlag GmbH Germany, part of Springer Nature 2020

I read with great interest the article written by Choi et al., "Potential benefits of salvage intratympanic dexamethasone injection in profound idiopathic sudden sensorineural hearing loss [1]." The authors compared the hearing recovery levels after initial treatment or salvage intratympanic dexamethasone injection (ITDI) and evaluated the prognostic factor on salvage ITDI therapy in profound idiopathic sudden sensorineural hearing loss. However, there were potentially incorrect data in the article.

First, for "Sex (M:F)" of Table 1, the ratios "47:56" for initial treatment group and "55:60" for salvage group should be misplaced and the two ratios should be exchanged for their places, as shown in this table "Initial treatment group $(n=115)$ " and "Salvage group $(n=103)$ ".

Second, for "Initial PTA (dB HL)" of Table 1, there should be decimal point misplacement for the number "10.725", which should be "107.25", and the corrected data "107.25 \pm 10.26 " and the data " $108.13 \pm 10.01$ " should be exchanged for their places, based on the description "In the initial-treatment group, the mean PTAs before and after initial treatment were $107.25 \pm 10.26$ and $87.07 \pm 25.73 \mathrm{~dB}$, respectively", in lines $10-12$ of the right column on page 3 and also the labeled number "107.25" for "Initial PTA" on the first bar of Fig. 2.

Third, the labeled numbers "93.02" on the second bar and "87.07" on the third bar of Fig. 2 were misplaced and the two numbers should be exchanged for their places, as the authors' description "In the initial-treatment group, the mean

This comment refers to the article available online at https://doi. org/10.1007/s00405-020-05967-z.

Entong Wang

wang_entong@163.com

1 Department of Otolaryngology-Head and Neck Surgery,

Beijing Electric Power Hospital, A1 Taipingqiao Xili,

Beijing 100073, China
PTAs before and after initial treatment were $107.25 \pm 10.26$ and $87.07 \pm 25.73 \mathrm{~dB}$, respectively. In the salvage group, the mean PTAs before and after salvage ITSI therapy were $93.02 \pm 17.88$ and $72.18 \pm 25.05 \mathrm{~dB}$, respectively", in lines $10-15$ of the right column on page 3 .

It seems that some of data in Table 1 and Fig. 2 are incorrect. Therefore, we recommend that the authors correct any possible errors in the article.

Author contributions All authors contributed to manuscript writing and approved the final manuscript.

Funding The authors did not receive any grants from any funding agencies.

\section{Compliance with ethical standards}

Conflict of interest The authors declare that they have no conflict of interest.

\section{Reference}

1. Choi JW, Lee CK, Kim SB et al (2020) Potential benefits of salvage intratympanic dexamethasone injection in profound idiopathic sudden sensorineural hearing loss. Eur Arch Otorhinolaryngol 277(8):2219-2227. https://doi.org/10.1007/s00405-020-05967 $-\mathrm{Z}$

Publisher's Note Springer Nature remains neutral with regard to jurisdictional claims in published maps and institutional affiliations. 